\title{
USE OF MAGNESIUM SULFATE IN CONTINUOUS INFUSION IN PATIENTS WITH SEVERE ACUTE ASTHMA, IN A PEDIATRIC EMERGENCY ROOM
}

\author{
Milton Gross Júnior ${ }^{1}$, Patricia Miranda Lago ${ }^{1}$, João Carlos Santana ${ }^{1}$, Gabriela Biondo ${ }^{2}$, \\ Bianca Zandona ${ }^{2}$, Fernanda Chiaradia ${ }^{2}$, and Paulo Roberto Carvalho ${ }^{1}$ \\ ${ }^{1}$ Universidade Federal do Rio Grande do Sul \\ ${ }^{2}$ Hospital de Clinicas de Porto Alegre
}

July 29, 2020

\begin{abstract}
Introduction: Asthma is a disease with important morbidity and that can lead to death in childhood. The use of intravenous magnesium sulfate has been indicated in cases refractory to the initial management with inhaled bronchodilators and corticosteroids. Objective: To evaluate the use of magnesium sulfate in continuous infusion $(50 \mathrm{mg} / \mathrm{kg} / \mathrm{hour}$ in 4 hours) in children with severe acute asthma. Location: 10-bed pediatric emergency room, university hospital. Patients: Children over 2 years old who received a continuous infusion of magnesium sulfate at a dose of $50 \mathrm{mg} / \mathrm{kg} / \mathrm{hour}$ in 4 hours. Methods and main findings: Cross-sectional, prospective study. All patients with severe acute asthma were included in a study protocol. A total of 40 patients met the inclusion criteria, $60 \%$ male, with a median age of 3.0 years (2.8-4.3). All patients were monitored and followed by an emergency pediatrician during the 4 hours of infusion. There was no description of adverse events related to the magnesium sulfate. The modified Wood-Downes clinical score was applied and compared before and after the infusion and a significant clinical improvement was observed $(\mathrm{p}<0.001)$. The serum magnesium levels at the end of the infusion ranged from $3.3-5.8 \mathrm{mg} / \mathrm{dL}$, suitable as therapeutic and without toxicity (median 4.0). The median length of stay in pediatric emergency was 2 days. Only 2 patients (5\%) were transferred to the PICU. Conclusions: On this study, the use of continuous magnesium sulfate proved to be well tolerated, leading to improved respiratory status, and can be considered as adjunctive therapy in the management of severe asthma.
\end{abstract}

USE OF MAGNESIUM SULFATE IN CONTINUOUS INFUSION IN PATIENTS WITH SEVERE ACUTE ASTHMA, IN A PEDIATRIC EMERGENCY ROOM

Milton Gross, Patricia M Lago, Fernanda Chiaradia, Bianca Zandoná, Gabriela Biondo, João Carlos Santana, Paulo Roberto Carvalho

Summary:

Introduction: Asthma is a disease with important morbidity and that can lead to death in childhood. The use of intravenous magnesium sulfate has been indicated in cases refractory to the initial management with inhaled bronchodilators and corticosteroids.

Objective: To evaluate the use of magnesium sulfate in continuous infusion ( $50 \mathrm{mg} / \mathrm{kg} /$ hour in 4 hours) in children with severe acute asthma.

Location: 10-bed pediatric emergency room, university hospital.

Patients: Children over 2 years old who received a continuous infusion of magnesium sulfate at a dose of $50 \mathrm{mg} / \mathrm{kg} / \mathrm{hour}$ in 4 hours. 
Methods and main findings: Cross-sectional, prospective study. All patients with severe acute asthma were included in a study protocol. A total of 40 patients met the inclusion criteria, $60 \%$ male, with a median age of 3.0 years (2.8-4.3). All patients were monitored and followed by an emergency pediatrician during the 4 hours of infusion. There was no description of adverse events related to the magnesium sulfate. The modified Wood-Downes clinical score was applied and compared before and after the infusion and a significant clinical improvement was observed $(\mathrm{p}<0.001)$. The serum magnesium levels at the end of the infusion ranged from $3.3-5.8 \mathrm{mg} / \mathrm{dL}$, suitable as therapeutic and without toxicity (median 4.0). The median length of stay in pediatric emergency was 2 days. Only 2 patients (5\%) were transferred to the PICU.

Conclusions: On this study, the use of continuous magnesium sulfate proved to be well tolerated, leading to improved respiratory status, and can be considered as adjunctive therapy in the management of severe asthma.

Introduction:

Asthma is the most common chronic disease in childhood, affecting about $10 \%$ of all children, being responsible for a large number of visits to the pediatric emergency $(1,2)$. Severe acute asthma is defined as an asthma attack that does not respond to conventional anticholinergic therapy, repeated doses of beta-agonists and corticosteroids, requiring hospitalization (3). There are some options for a second treatment scale, one of which is magnesium sulfate, intravenously $(4,5)$.

Magnesium sulfate is a drug that affects both the muscles of the airway and reduces the inflammatory process. Its mechanism of action is due to its spasmolytic properties, that is, the serum ionized magnesium above the physiological levels produces a transient blockage of the calcium channels regulated by the N-methylD-aspartate receptor, generating muscle relaxation. Blocking the entry of calcium into the smooth muscle of the airway interferes with the contraction of the smooth muscle, inducing bronchodilation. In addition, magnesium sulfate has an effect in inhibiting the release of acetylcholine by the motor nerve terminals, inhibiting the release of histamine from mast cells, thus decreasing the production of mucus by the secretory glands (6-9).

Several studies have suggested the use of magnesium sulfate for the treatment of severe acute asthma attacks, in children who did not respond to standard treatment, in a single dose $(25-100 \mathrm{mg} / \mathrm{kg})$, intravenously and in bolus. However, despite having a rapid peak of action, its spasmolytic effect decreases abruptly due to rapid renal elimination, even when administered at high doses (12-14).

As a strategy to maintain a consistent therapeutic serum level to compensate for rapid renal elimination, some researchers have proposed the continued use of magnesium sulfate intravenously (14-20). In a recent study carried out by Irazuzta and collaborators, they demonstrated that the use of magnesium sulfate, at a dose of $50 \mathrm{mg} / \mathrm{kg} / \mathrm{hour}$ for 4 hours when compared to bolus treatment $(50 \mathrm{mg} / \mathrm{kg} /$ hour in 1 hour), had better results in the time of disease progression, improvement in lung function, reduction in the hospitalization rate and a better cost-benefit ratio (17). Likewise, Torres, studying patients in a pediatric ICU, described that the infusion of magnesium sulfate during the first hour of hospitalization in patients with severe asthma significantly reduced the percentage of children who required mechanical ventilation (18).

The aim of this study was to evaluate the use of continuous magnesium sulfate over 4 hours in patients with severe acute asthma, who were admitted to the pediatric emergency room by varying the clinical response of the serum magnesium level and the presence of adverse events after infusion of medication.

1. Methods:

2. Study design and location:

This cross-sectional, observational, prospective study was submitted to the Ethics Committee and was accepted and filed under the number 23635618.1.0000.5327. We evaluated all patients with severe acute asthma who received magnesium sulfate in continuous infusion, admitted to the pediatric emergency room in the period from April 2017 to October 2019. Patients at the time of magnesium sulfate prescription were included in the study protocol where the medical record number and medication administration date were 
recorded for future review of data from the electronic medical record. All children eligible for the study remained monitored and accompanied by emergency pediatricians during the infusion. The prescription of magnesium sulfate was the responsibility of the attending physician, following the care protocol of the unit. Serum magnesium was collected immediately after the end of the infusion.

Selection of patients:

All patients over 2 years of age with acute severe asthma who received magnesium sulfate in continuous infusion at a dose of $50 \mathrm{mg} / \mathrm{kg} /$ hour for 4 hours were included. Patients with a previous history of nephropathy, heart disease, pneumopathies and genetic syndromes associated with pulmonary impairment were excluded. Also excluded were those who used the medication more than once and those who used other intravenous bronchodilator drugs concurrently.

Demographic data and outcomes:

Data were placed in a Microsoft Excel spreadsheet. The variables evaluated were age, gender, weight, height, serum magnesium dosage, presence of adverse events, presence of pneumonia, use of antibiotics, clinical evolution through the modified Wood-Downes severity score (picture 1). The outcomes analyzed were length of stay in the pediatric emergency, need for admission to a pediatric ward or PICU, use of ventilatory support and death $(21,22)$.

\begin{tabular}{lll}
\hline Clinical parameters & 0 & 1 \\
\hline Skin color & Normal & Pallor \\
Respiratory frequency (ipm) 2-5 years $>5$ years & Up to 25 Up to 20 & $26-5021-40$ \\
Use of accessory musculature & None & Mild or moderate \\
Pulmonary auscultation & Wheezing at the end of inspiration & Inspiratory and expiratory wheezin \\
Brain function & Normal & Depressed or restless \\
\hline
\end{tabular}

Picture 1: Clinical score for assessing the intensity of asthma attacks in children (modified Wood-Downes score) (22).

Analysis:

The data were analyzed using the SPSS software, version 16.0. Categorical variables were expressed as a percentage and compared using the Chi-square test or relative risk. Continuous variables with normal distribution were expressed using means with the respective standard deviation and compared using Student's $t$ test. Continuous variables without normal distribution were expressed using the median with the respective interquartile range (IQ 25\% -75\%) and compared using the Mann-Whitney or Kruskal-Wallis tests. Correlation tests were also performed (Pearson's correlation coefficient and determination coefficient).

Results: 


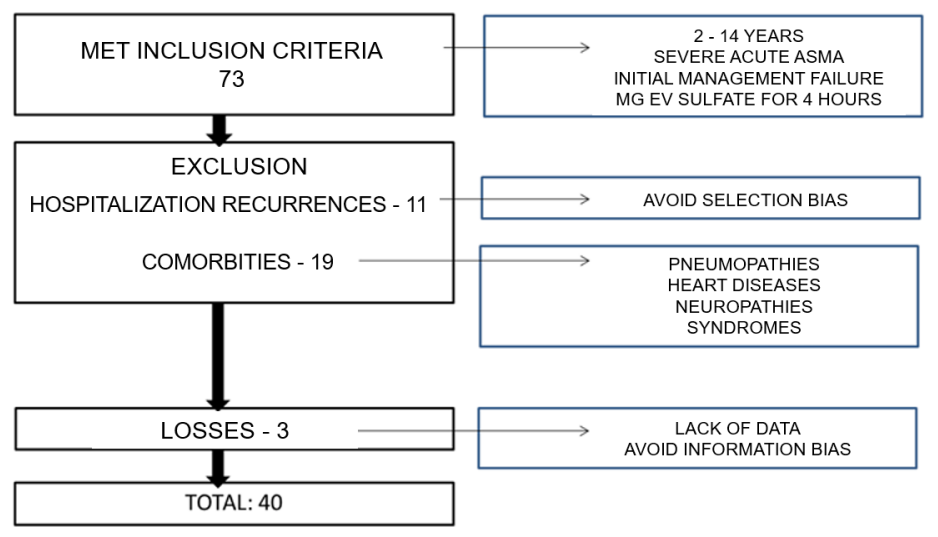

Picture 2: Flowchart of the study population.

During the study period, 73 patients met the inclusion criteria. Of these, 11 patients were excluded due to recurrence of hospitalizations or the need for a second dose of magnesium sulfate. In a second analysis, 19 of the remaining 62 patients were excluded due to the presence of comorbidities. There was a loss of 3 patients due to the lack of information in the electronic medical record, ending in a total of 40 patients, according to a previous sample calculation.

Of the 40 patients, 24 were male (60\%) and 16 female (40\%), with a median age of 3 years (2.8-4.3). By calculating BMI, no patient was overweight or obese, with a median weight of $18 \mathrm{~kg}$ (14-23.5).

Through the review of electronic medical records, it was possible to classify patients according to the modified Wood-Downes severity score (picture 1). Before the continuous magnesium sulfate infusion for 4 hours, a variation in the score value from 3 to 6 was observed, with a median of 4 (3-5). After 4 hours of magnesium sulfate administration, the scale values decreased to a range from 5 to 0 with a median of $1(0-2)$, showing a statistically significant difference $(\mathrm{p}<0.001)$ (picture 3$)$.

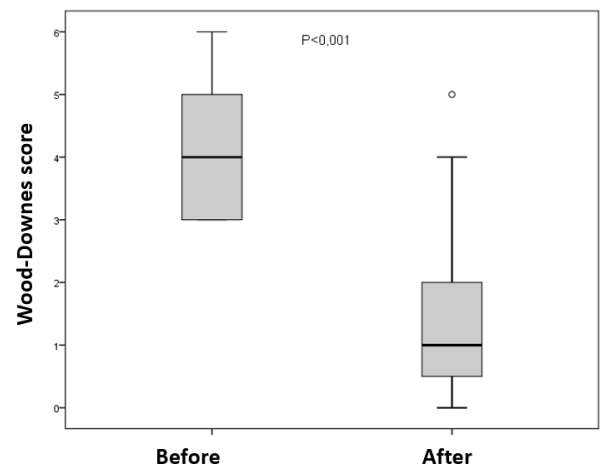

Picture 3: Correlation of the Wood-Downes clinical score before and after 4 hours of continuous magnesium sulfate infusion. 
The serum magnesium level collected after 4 hours of continuous infusion varied from 3.3-5.6 mg/dL, with a median of $4.3(3.9-4.6)$.

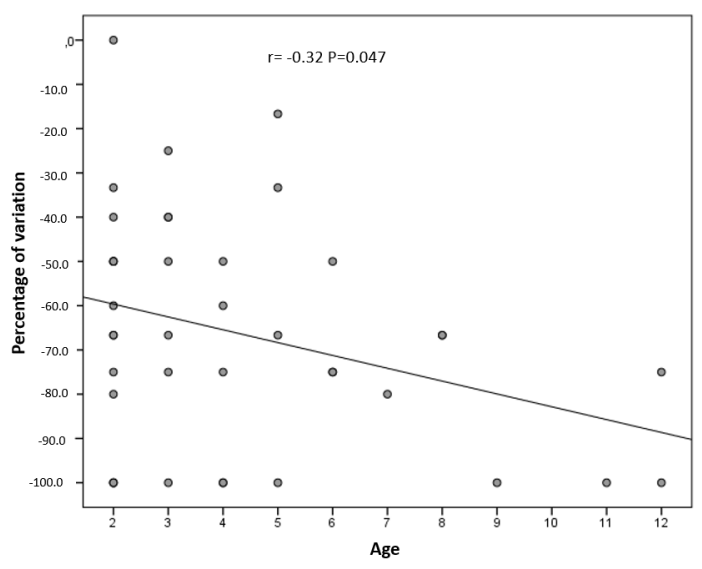

Picture 4: Correlation of the percentage of variation of the Wood-Downes clinical score with age in years.

As we can see in Picture 4, there was a correlation between the percentage of variation of the clinical score with age $(\mathrm{p}=0.047)$.

The presence of pneumonia was considered through the report of the chest radiography with the description of foci of consolidation. Of the 40 patients studied, only $9(22.5 \%)$ had a radiological picture of pneumonia, however, 15 (37.5\%) patients received antibiotics. Among the main drugs prescribed were: Azithromycin (14), Amoxicillin (3), Ampicillin (2) and Cefuroxime (2). There was no relationship between the presence of pneumonia and the non-response to the use of intravenous magnesium sulfate $(\mathrm{p}=0.969)$, just as there was no relationship between the use of antibiotics and the non-response to the use of intravenous magnesium sulfate $(\mathrm{p}=0.965)$.

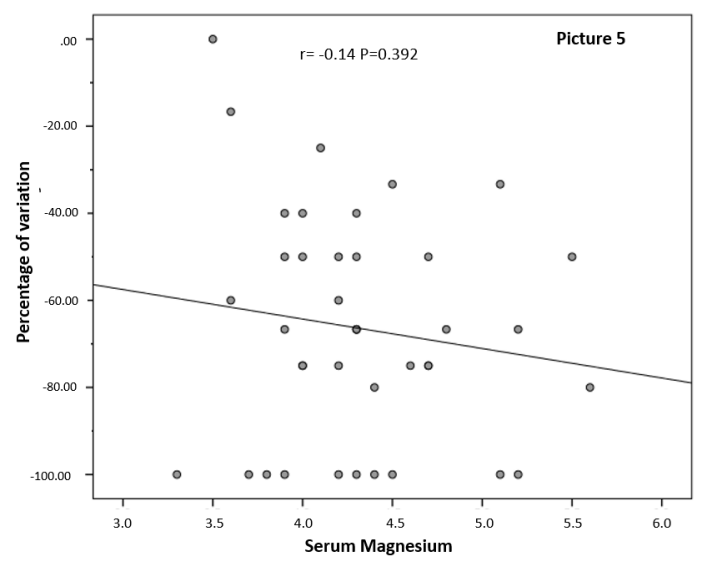

Picture 5: Correlation of the variation percentage of the Wood-Downes clinical score with the serum magnesium level at the end of the 4-hour continuous infusion of magnesium sulfate. 
When comparing the serum magnesium level and a better clinical response, assessed by varying the score ( $\mathrm{p}$ $=0.392)$, no correlation was observed. Using a cutoff point of serum magnesium level at 4.0, as suggested in the literature as a therapeutic mean, no significant variation was observed $(\mathrm{p}=0.77)$ (Picture 5).

There were no reports of any adverse events during and after the intravenous magnesium sulfate infusion in 4 hours, researching the description of nausea, drowsiness, vision changes, muscle weakness, hypotension, respiratory distress, arrhythmia and burning sensation and redness in the application route.

The average length of stay in the pediatric emergency was 2 days. Only $2(5 \%)$ patients required transfer to the PICU, but without the need for ventilatory support. There was no correlation between the length of stay in the pediatric emergency and the variation in the clinical score $(\mathrm{p}=0.242)$. None of the patients in the study died.

Discussion:

The management of asthma in the emergency room must be fast and organized due to the reversibility possibility of the condition and the potential risk of progression to respiratory failure (3). The classic treatment includes repeated inhaled beta-adrenergics and corticosteroids. In acute and severe cases, magnesium sulfate has been used intravenously after failure to respond to initial management. Magnesium produces smooth muscle relaxation inducing bronchodilation and has a rapid peak of action, but is also rapidly eliminated by the kidney, which restricts its use. In order to prolong the effect of magnesium sulfate, maintaining a compatible therapeutic level to compensate for the rapid elimination, Irazuzta et al. suggested the continuous infusion of this medication $(16,19,20)$.

The present study evaluated children who were admitted to the pediatric emergency room with severe acute asthma and who used a continuous infusion of magnesium sulfate for 4 hours, at a dose of $50 \mathrm{mg} / \mathrm{kg} / \mathrm{hour}$. It was possible to observe that there was a significant clinical improvement after the end of the infusion, the serum magnesium level ranged from 3.3 to $5.6 \mathrm{mg} / \mathrm{dL}$, very close to the levels described in the literature as therapeutic (around $4.0 \mathrm{mg} / \mathrm{dL}$ ). No patient experienced adverse events (8).

The beneficial effect of magnesium sulfate on asthma is well known, but few studies have been conducted in children. In a 2016 Cochrane review, only 5 studies with a more robust methodology were identified, where the authors concluded that intravenous magnesium sulfate could reduce the need for hospital admission in patients with moderate to severe asthma, refractory to the initial treatment. In our study, a significant clinical improvement was observed after 4 hours of infusion, assessed by varying the clinical severity score and only 2 out of 40 patients did not respond to magnesium sulfate, even with an adequate serum level, requiring transfer to PICU (16).

The normal concentration of serum magnesium in humans is 1.8 to $2.3 \mathrm{mg} / \mathrm{dL}$. It is the fourth most abundant mineral in the body, it acts as a cofactor in more than 300 enzymatic reactions, it is necessary for the synthesis of proteins, including DNA and RNA. The ideal level of serum magnesium for the treatment of wheezing exacerbations in childhood is not well established in the literature. Several studies have reported achieving therapeutic efficacy with serum concentrations between 3 and $5 \mathrm{mg} / \mathrm{dL}$, corroborating the data found in this study in which the median serum magnesium was 4.3 (3.9-4.6) after 4 hours of infusion (13-20).

The toxicity of magnesium sulfate has already been well evidenced in obstetric and anesthetic studies, mainly associated with the treatment of eclampsia, however there is little description outside these contexts. The side effects of magnesium sulfate are known to be dose dependent. Symptoms such as nausea, flushing, drowsiness, changes in vision and muscle weakness are more common when serum magnesium concentrations exceed 9 $\mathrm{mg} / \mathrm{dL}$. On the other hand, Graft and collaborators observed that the maintenance of serum magnesium levels between 4 and $6 \mathrm{mg} / \mathrm{dL}$ are effective in avoiding clinical signs of toxicity (15). From the data found in this study, we can observe that the use of magnesium sulfate by continuous infusion in 4 hours at a dose of $50 \mathrm{mg} / \mathrm{kg} /$ hour raises the serum magnesium level to therapeutic values, non-toxic however $(14,15,19)$.

Our study has limitations because it is a case series without a control group. Associated with this, it is a review of electronic medical records, subject to some interpretation bias, mainly in the subjective 
data described, however we try to minimize this fact by using only one reviewer researcher. Despite these restrictions, the results obtained corroborate with the data presented in the literature so far, showing that magnesium sulfate in continuous infusion could be useful in the treatment of severe acute asthma safely. New studies, ideally multicentric, randomized and with a larger sample size, can contribute to a deeper knowledge on the subject.

Conclusion:

Based on this study, we concluded that the use of continuous intravenous magnesium sulfate at a dose of $50 \mathrm{mg} / \mathrm{kg} /$ hour in 4 hours can be a satisfactory adjuvant therapy in the setting of severe acute asthma in children. Associated with the advantage of a good clinical response, magnesium sulfate was shown to be safe, maintaining a non-toxic and therapeutic serum level, with no adverse events observed.

References:

1. Global Initiative for Asthma [homepage on the Internet]. Bethesda: Global Initiative for Asthma [cited 2016 Jun 07]. Global Strategy for Asthma Management and Prevention 2016. [Adobe Acrobat document, 151p.]. Available from: http://ginasthma.org/wp-content/).

2. Eder W, Ege MJ, von Mutius E. The asthma epidemic. N Engl J Med. 2006;35: 2226-2235.

3. Shein S, Specher H, Proença JP et al. Tratamento atual da criança com asma critica quase fatal. Ter. Bras. Ter. Int ;2016:167-178.

4. Solé D, Rosário Filho NA, Sarinho E et al.. Prevalence of asthma and allergic diseases in adolescents: nine-year follow-up study (2003-2012). J Pediatr (Rio J). 2015; 9:30-35.

5. Ciarallo L; Sauer A, Shannon M et al. Intravenous magnesium therapy for moderate to severe pediatric asthma: Results of a randomized, placebo-controlled trial. J Pediatrics 1996; 129:.809-814.

6. Cairns, 1996 Cairns CB, Krafi M. Magnesium attenuates the neutrophil respiratory burst in adult asthmatic patients. Academic Emergency Medicine 1996: 3:1093-1097.

7. Spivey, 1990 Spivey WH, Skobeloff EM, Levin RM. Effect of magnesium chloride on rabbit bronchial smooth muscle. Annals of Emergency Medicine 1990:19:1107-1112.

8. Glover ML, Machado C, Totapally BR: Magnesium sulfate administered via continuous intravenous infusion in pediatric patients with refractory wheezing. J Crit Care 2002: 17:255-258 .

9. Goodacre S, Cohen J, Bradburn M, et al; 3Mg Research Team: The 3Mg trial: A randomised controlled trial of intravenous or nebulised magnesium sulphate versus placebo in adults with acute severe asthma. Health Technol Assess 2014; 18:1-168 .

10. Singhi S, Grover S, Bansal A, et al: Randomised comparison of intravenous magnesium sulphate, terbutaline and aminophylline for children with acute severe asthma. Acta Paediatr 2014; 103:13011306 .

11. Cairns, 1996 Cairns CB, Krafi M. Magnesium attenuates the neutrophil respiratory burst in adult asthmatic patients. Academic Emergency Medicine 1996;3:1093-1097.

12. Chesley LC, Tepper I: Some effects of magnesium loading upon renal excretion of magnesium and certain other electrolytes. J Clin Invest 1958; 37:1362-1372.

13. Rower J, Liux Y. Clinical pharmacokinetics of magnesium sulfate in the treatment of children with severe acute asthma. Eur. J. Pharmacol. 2017;73:325-331.

14. Egelund T. High-dose magnesium sulfate infusion protocol for status asthmaticus: a safety and pharmacokinetics cohort study. Intensive Care Med 2013; 39: 117-122.

15. Graft D. Stevenson M, Berkenbosch J. Safety of prolonged magnesium sulfate infusion during treatment for severe pediatric status asthmaticus. Pediatric Pulmonology 2019; 51:1-7.

16. Griffiths B, Kew KM. Intravenous magnesium sulfate for treating children with acute asthma in the emergency department. Cochrane Database of Systematic Reviews 2016, Issue 4. Art. No.: CD011050).

17. Irazuzta J. Paredes F, Pavlivich V. High-Dose Magnesium Sulfate Infusion for Severe Asthma in the Emergency Department: Efficacy Study. Pediatr Crit Care Med 2016; 17: 29-33.

18. Torres S. Effectiveness of magnesium sulfate as initial treatment of acute severe asthma in children, conducted in a tertiary-level university hospital: a randomized, controlled trial. Arch Argent Pediatr 
2012: 110; 291-296.

19. Irazusta JE, Chiriboga N. Magnesium Sulfate infusion for acute asthma in the emergency department. J Pediatr (Rio J) 2017; 93:19-25.

20. Pruikkonen H, Tapiainen T, Kallio M, et al. Intravenous magnesium sulfate for acute wheezing in young children: a randomised double-blind trial. Eur Respir J 2018; 51: 1701579 [https://doi. org/10.1183/13993003.01579-2017.

21. Wood, D. Downes J, Leckes H. A clinical scoring system for the diagnosis of respiratory failure. Preliminary report on childhood status asthmaticus. Am J Dis Child 1972; 123: 227-228.

22. Paro, ML; Rodrigues JC. Factors predictive of the development of acute asthma attacks in children. J Bras Pneumol 2005;31: 373-381, 2005. 